\title{
An ionic bottle for high-speed, long-retention memristive devices
}

\author{
Dmitri B. Strukov • R. Stanley Williams
}

Received: 1 November 2010 / Accepted: 22 December 2010 / Published online: 16 February 2011

(c) The Author(s) 2011. This article is published with open access at Springerlink.com

\begin{abstract}
We propose a new type of bipolar resistive switch or memristive device, based on an ionic bottle, which is characterized by both low switching energy and high state retention time. The ionic bottle is engineered such that image charges on metallic electrodes trap ions inside a semiconducting film when there is no applied bias voltage, but an external field can drive ions through one of the electrodes into an adjacent semiconducting film to accomplish a nonvolatile resistance change.
\end{abstract}

A promising device for 'universal' memory and storage is the bipolar resistance switch, of which there are now many known examples [3, 8]. Recently, we demonstrated [5] that such switches were physical examples of the memristor [1] or memristive device [2], which were introduced as a new fundamental passive two-terminal circuit element. An essential issue regarding these devices is the "pinched hysteresis loop", i.e. the observation that the current/voltage $(I-V)$ characteristic always passes through the origin for any periodic applied excitation. This property means that the device does not store charge or energy, but it does store information; the value of the resistance of the device at time $t$ is a function of the total integrated current (equivalently, the total charge) that has passed through the device. Thus, an ideal memristor should be nonvolatile; it should be able to hold its state indefinitely. However, since the mechanism for real

D.B. Strukov $(\bowtie)$

University of California Santa Barbara, Santa Barbara, CA 93106,

USA

e-mail: strukov@ece.ucsb.edu

\section{R.S. Williams}

Hewlett-Packard Laboratories, 1501 Page Mill Road, MS1123,

Palo Alto, CA 94304, USA bipolar switches often involves the drift of charged species under the influence of an applied electric field [5, 9], the actual state lifetime of a device may be limited by the diffusion of the mobile species back to their original configuration.

For example, the complete and partial OFF states for a thin-film memristive device simulated in Ref. [7] are not thermodynamically stable. After the device is switched to the OFF state and the external bias is removed the mobile ions will slowly diffuse toward the middle of the film, turning the device back to the ON state. The rate of such relaxation can be determined by plotting the zero-bias conductance as a function of time (black line in Fig. 1). The absolute value of the relaxation rate, which depends on device geometry, mobility $\mu_{\mathrm{I}}$ and diffusivity $D_{\mathrm{I}}$ of the ions in the film, does not conveniently reflect the retention characteristics of the device. Instead, it is more useful to consider the ratio of the relaxation rate, equivalent to the retention time $\tau_{\text {store }}$, with respect to the time required to turn the device from the OFF state to the ON state by applying a constant external bias $v$ (of the polarity opposite to that used to turn the device to the OFF state), i.e. the time $\tau_{\text {write }}$ to write

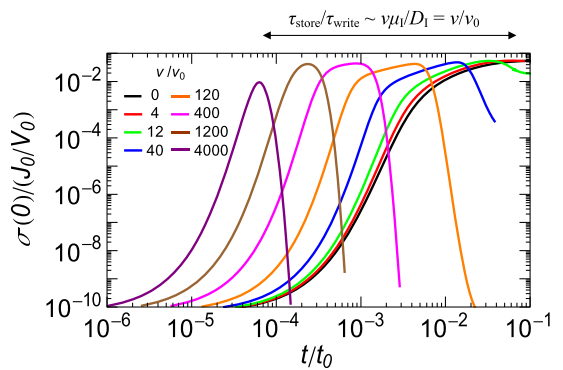

Fig. 1 Zero-bias conductance evolution as a function of time for several external biases calculated using the model considered in Ref. [6]. Here all axes are dimensionless with $t_{0}, J_{0}$, and $v_{0}$ constants defined similarly to Ref. [6] 


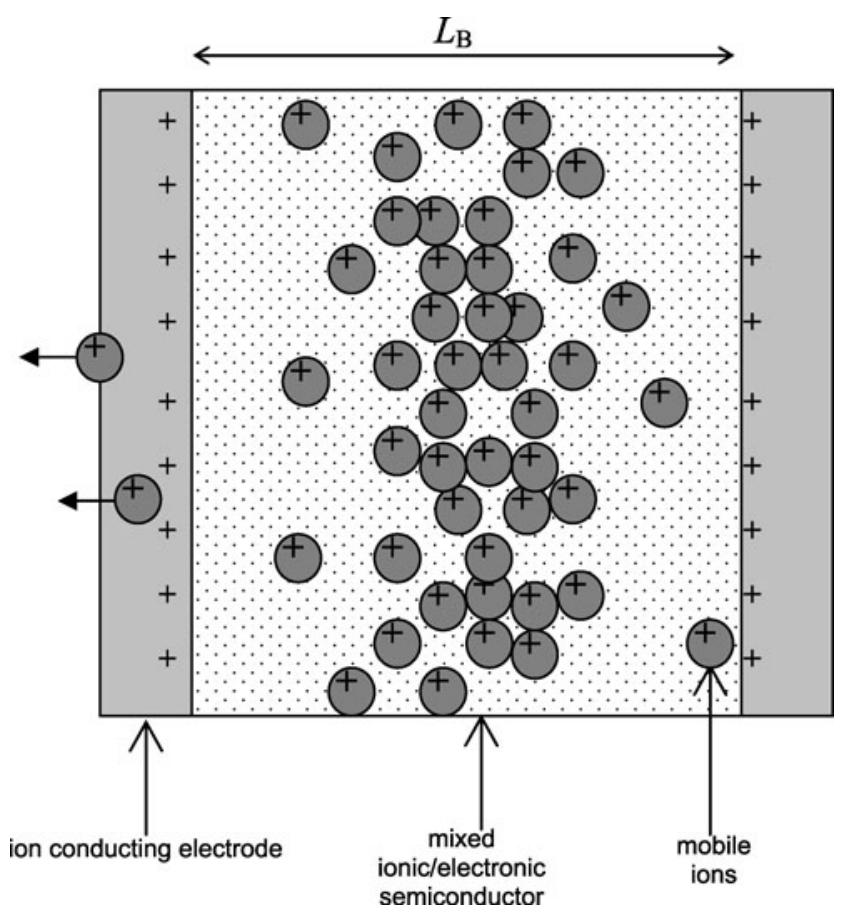

Fig. 2 "Ionic bottle" effect

the device. For the model considered in Ref. [6], a bias $v$ applied to an OFF state device first turns the device to the ON state and then to a second OFF state (i.e., characterized by the reversed ionic concentration profile), and $\tau_{\text {write }}$ is determined by the peak position of zero-bias conductance (Fig. 1). When only linear ionic drift is considered, the ratio of $\tau_{\text {store }} / \tau_{\text {write }}$ is not large (generally less than 1000) and clearly proportional to $v / v_{0}$ [7] (here $v_{0}=e / k_{\mathrm{B}} T$ is the characteristic thermal voltage, where $e, k_{\mathrm{B}}, T$ are the electron charge, the Boltzmann constant and the temperature, respectively).

The retention characteristics could be dramatically improved by choosing an appropriate mobile species and host material (e.g. positively charged oxygen vacancies that act as dopants in $\mathrm{TiO}_{2}$ ). Because of internal Joule heating during switching and the exponential dependence of the drift velocity at very high electric fields [7], it is possible to obtain a large dynamic ratio between switching time and state retention time (as many as 15 orders of magnitude). However, this requires the existence of particular and perhaps rare materials properties. In this paper, we describe a more general structure, the ion bottle, which has the potential to realize bipolar memristance with low power dissipation, high switching speed and long state retention time that depends only weakly on materials properties.

Consider a semiconductor film of thickness $L_{\mathrm{B}}$ sandwiched between two conductive electrodes and containing a significant concentration of charged mobile species, e.g. positive $\mathrm{O}$ vacancies in $\mathrm{TiO}_{2}$ (Fig. 2). In general, we are interested in keeping the ions inside the semiconductor film

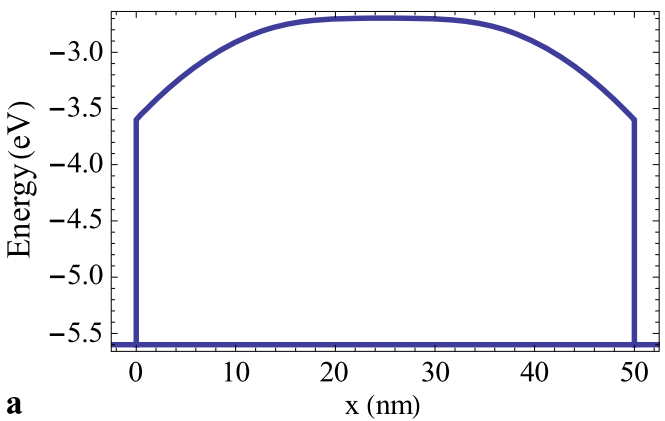

$\mathbf{a}$

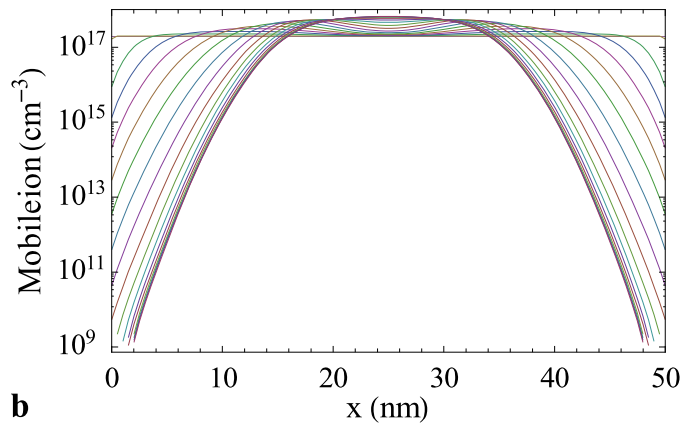

Fig. 3 Equilibrium mobile-ion concentration profile based on the self-consistent simulation model described in Ref. [6]. Panel (a) shows the bending of the bottom of the conduction band with energy levels corresponding to that of rutile $\mathrm{TiO}_{2}$, while (b) shows the relaxation of the mobile ions starting from a uniform concentration $n(x)=2 \times 10^{17}$. The background acceptor doping is $3 \times 10^{18} \mathrm{~cm}^{-3}$, while the energy levels of the donors and acceptors are assumed to be equal to the bottom of the conduction band and the top of the valence band, respectively

even if the electrodes are permeable (e.g. metallic oxides such as $\mathrm{RuO}_{2}$ or $\mathrm{IrO}_{2}$ ) and the concentration of mobile dopants outside the device is negligible. To achieve this goal, assume that the bulk Fermi level of the semiconductor layer is lower than that of the electrodes by the value $U$. This could be due to additional doping of the semiconductor with immobile acceptors (e.g. $\mathrm{C}$ or $\mathrm{N}$ in $\mathrm{TiO}_{2}$ ) or to the intrinsic alignment between the electrochemical potentials of the semiconductor (e.g. $\mathrm{WO}_{3}$ ) and the metallic electrodes. In this ionic bottle, the electrodes are positively charged and repel the positively charged mobile ions toward the middle of the device. The mobile ionic concentration profile at equilibrium resembles an upside down $U$. (Alternatively, for negatively charged acceptor-like mobile dopants, the electrodes could be negatively charged if the Fermi energy of the semiconductor is above that of the electrode metal.) Figure 3 shows self-consistent numerical simulation results for the steady-state solution of the Boltzmann-Poisson equations assuming Fermi-Dirac statistics for the electron distribution inside semiconductor layer [6].

Neglecting the space charge effects of the mobile dopants (which is valid if the background immobile acceptor doping is much larger), the dependence of the mobile-ion concen- 
tration $n(x)$ on the potential $\varphi$ near the electrode is given by the Poisson-Boltzmann equation:

$n(x) \approx N_{0} \exp \left[e \varphi(x) /\left(k_{\mathrm{B}} T\right)\right]$,

where $N_{0}$ is a constant that is determined by the normalization condition $\int_{0}^{L} n(x) d x=N_{\text {total }}$. When the voltage across the device is zero, the concentrations of mobile dopants near the interfaces are close to $N_{0}$. Assuming that the concentration of mobile ions outside the metallic electrodes is negligibly small, the rate of escape of ions from the volume is proportional to the ion diffusion current

$J_{\mathrm{diff}} \approx e D_{\mathrm{M}} N_{0} / a$

where $a$ is the lattice constant and $D_{\mathrm{M}}$ is the diffusion constant of the ions in the electrode material. When an external positive voltage $V$ is applied to the right electrode of the device, the mobile-ion concentration near the left electrode/semiconductor interface increases exponentially. The ratio of ion escape rates from the device when the applied voltage changes from zero to $V$ is

$R=J_{\text {diff }}(v=0) / J_{\text {diff }}(v=V)=\exp \left[e V /\left(2 k_{\mathrm{B}} T\right)\right]$.

For $T=300 \mathrm{~K}, R$ can be as high as $10^{17}$ at $V=2 \mathrm{~V}$ and $10^{9}$ at $1 \mathrm{~V}$, and is independent of the kinetic properties of the device.

This exponential modulation of the mobile-ion concentration near the electrodes by an external voltage bias, and the resulting exponential change of the rate of inward or outward ionic currents is the main result of this paper. We now describe an example of a memristive device based on this ionic bottle effect (Fig. 4). The device consists of two semiconductor films connected by a permeable metal electrode and confined by two impermeable outer metallic electrodes (e.g. Pt). The right semiconductor film of the device is assumed to be heavily doped with immobile acceptors with a uniform concentration $N_{\mathrm{A}}{ }^{(\mathrm{B})}$, which is much larger than that of the mobile ions so that the electrical resistance of this film is independent of small changes in the mobile dopant concentration. The left film of the device is also doped with immobile acceptors, but the smaller concentration $N_{\mathrm{A}}{ }^{\text {(D) }}$ is such that the presence of mobile dopants significantly changes the resistance of the device. Here, we assume that diffusive transport of electrons determines the resistance of the semiconductor films [6].

When no bias is applied to the device, the mobile ions are effectively confined in both layers and only redistribute between the two reservoirs with a rate determined by the concentration difference across the middle electrode, i.e. described by (2). Application of a voltage bias forces exponential changes in the concentrations near the two interfaces of the permeable electrode, and hence a dramatically faster
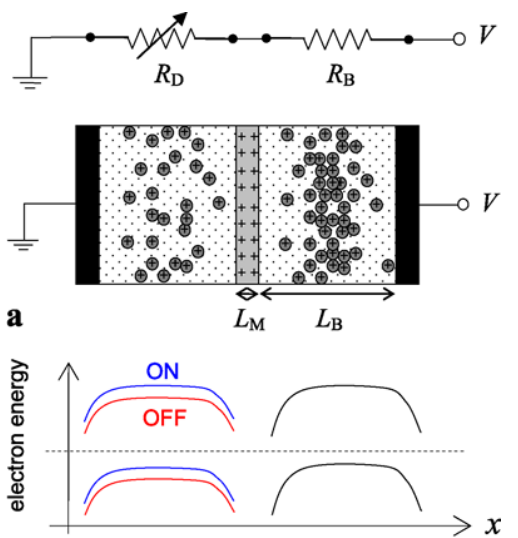

b

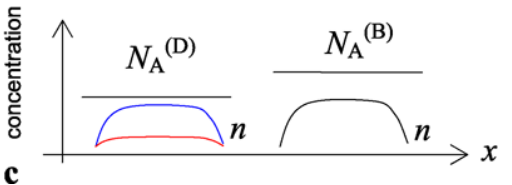

Fig. 4 An example of the memristive device: (a) cartoon of the device and equivalent circuit, (b) band diagram, and (c) mobile-ion concentration profile (schematically)

redistribution of ions with the direction depending on the bias polarity. Therefore, (3) effectively describes the ratio of state retention time to the switching time of the device.

We can estimate practical values of the parameters required to operate the device, and thus determine the plausible energy and speed performance. First, to suppress the concentration of mobile ions at the interface, a large band bending (built-in voltage) of the order of $1 \mathrm{eV}$ is required. The depletion width near the interface should be smaller than or comparable to the semiconductor film thickness. For the system shown in Fig. 3, the film thickness and doping should satisfy the condition $L_{\mathrm{B}} \approx\left(\varepsilon \varepsilon_{0} U k_{\mathrm{B}} T / N_{\mathrm{A}}{ }^{(\mathrm{B})}\right)^{1 / 2} / q$. Alternatively, $N_{\mathrm{A}}{ }^{(\mathrm{B})}$ could be replaced with $N_{\mathrm{D}} \times \exp \left[\Delta U_{\mathrm{gap}} /\left(k_{\mathrm{B}} T\right)\right]$, where $N_{\mathrm{D}}$ is the effective density of states at the appropriate edge of the band and $\Delta U_{\text {gap }}$ is the band gap of the semiconductor if no background doping is required to achieve sufficient band bending. For example, for $L=10 \mathrm{~nm}$, doping of the order $N_{\mathrm{A}}{ }^{(\mathrm{B})} \approx 5 \times 10^{18} \mathrm{~cm}^{-3}$ is needed (for a dielectric constant of the film $\varepsilon=10$ ).

For the two-terminal configuration described above, the metallic membrane is galvanically connected via the resistance of the right semiconductor film $R_{\mathrm{B}}$ to the right electrode. When a potential is applied across the full device, a portion of the voltage drops across the right hand side. Since $R_{\mathrm{B}}$ is much smaller than $R_{\mathrm{D}}$ (Fig. 4), this does not affect the on/off ratio of the device significantly. This could be achieved with nonlinear $I-V$ characteristics, i.e. if at low bias $R_{\mathrm{B}} \approx R_{\mathrm{D}}$ while at high biases $R_{\mathrm{B}} \ll R_{\mathrm{D}}$. Our previous work shows that in fact such nonlinearity might be achieved in the considered device structure due to the induced po- 
tential barrier for the electrons and/or holes caused by the dopant ion distribution [6]. On the other hand, there is no such limitation for a three-terminal configuration, for which an additional terminal is connected to the middle electrode, similar to the structure of a bipolar transistor. The threeterminal devices would be practical for applications that do not have a strict area density requirement and could accommodate more wires, such as an electrochromic device for a display application.

The three processes involved in mobile-ion redistribution when a bias voltage is applied are (1) establishing a new mobile dopant ion concentration at the permeable electrode interface, (2) diffusion of these ions through the electrode (the potential difference across the electrode is zero since it is metallic), and (3) redistribution of the ions in the volume of the target film. Since (2) should be the rate-limiting step, we can neglect (1) and (3) in the switching time estimate and thus $t_{\text {write }} \approx L_{\mathrm{M}}{ }^{2} / D_{\mathrm{M}}$. Obviously, the switching time will decrease by reducing the thickness of the permeable electrode, which should be thicker than the ThomasFermi screening length. For example, for few-nanometer membranes and $D_{\mathrm{M}}=10^{-5} \mathrm{~cm}^{2} \mathrm{~V}^{-1}$, the switching time could be less than 100 ns. Long state retention time and fast switching speed may be combined with small switching energy and currents. For $R_{\mathrm{D}}=1 \mathrm{G} \Omega, V=5 \mathrm{~V}$ and $t_{\text {write }}=100 \mathrm{~ns}$, the energy required to switch a bit should be less than $1 \mathrm{fJ}$, which is comparable with the best end of the roadmap semiconductor memories [4].

In summary, we proposed a device structure that can potentially combine high-speed switching $<100$ ns, low energy $\sim 1 \mathrm{fJ} /$ bit, and high state retention to a switching time ratio of $>10^{15}$. Moreover, the device operation is based on a linear mode for the ionic drift, which at the same time al- lows for high endurance because of the low energy (and thus Joule heating) required for device operation. In principle, the device can function with nanocrystalline or amorphous materials and therefore be compatible with backend processes enabling cost efficient multilevel integration.

Acknowledgement Work at UCSB is funded by NSF grant CCF1028336.

Open Access This article is distributed under the terms of the Creative Commons Attribution Noncommercial License which permits any noncommercial use, distribution, and reproduction in any medium, provided the original author(s) and source are credited.

\section{References}

1. L.O. Chua, Memristor - the missing circuit element. IEEE Trans. Circuit Theory 18, 507-519 (1971)

2. L.O. Chua, S.M. Kang, Memristive devices and systems. Proc. IEEE, 64(2), 209-223 (1976)

3. G. Dearnaley, A.M. Stoneham, D.V. Morgan, Electrical phenomena in amorphous oxide films. Rep. Prog. Phys. 33, 1129-1192 (1970)

4. International Technology Roadmap for Semiconductors 2009 Edition, available online at http://ww.itrs.net

5. D.B. Strukov, G. Snider, D. Stewart, R.S. Williams, The missing memristor found. Nature 453, 80-83 (2008)

6. D.B. Strukov, J.L. Borghetti, R.S. Williams, Coupled ionic and electronic transport model of thin-film semiconductor memristive behavior. Small 5(9), 1058-1063 (2009)

7. D.B. Strukov, R.S. Williams, Exponential ionic drift: Fast switching and low volatility of thin film memristors. Appl. Phys. A, Mater. Sci. Process. 94(3), 515-519 (2009)

8. R. Waser, M. Aono, Nanoionics-based resistive switching memories. Nat. Mater. 6, 833-840 (2007)

9. J.J. Yang, M.D. Pickett, X. Li, D.A.A. Ohlberg, D.R. Stewart, R.S. Williams, Memristive switching mechanism for metal/oxide/metal nanodevices. Nat. Nanotechnol. 3, 429-433 (2009) 